\title{
Nuclear effects on the $\rho$ meson produced in the inclusive photonuclear reaction
}

\author{
Swapan Das ${ }^{1}$ \\ Nuclear Physics Division, Bhabha Atomic Research Centre, \\ Trombay, Mumbai: 400085, India \\ Homi Bhabha National Institute, Anushakti Nagar, Mumbai-400094, India
}

\begin{abstract}
The hadronic properties of the $\rho$ meson produced in the inclusive photonuclear reaction have been investigated. The elementary reaction occurring in the nucleus is assumed as $\gamma N \rightarrow \rho^{0} N$. The $\rho$ meson, while propagating through the nucleus, interacts with the nuclear particles, and therefore, the properties of the $\rho$ meson can be modified because of this interaction. Being a short-lived particle, the $\rho$ meson decays to various elementary particles, such as, $e^{+} e^{-}, \pi^{+} \pi^{-}, \ldots$ etc. The $e^{+} e^{-}$ invariant mass, i.e., the $\rho$ meson mass, distribution spectra have been calculated to extract the information about the parameters, viz., mass and width, of the $\rho$ meson in the nucleus. The calculated results have been compared with the data reported from Jefferson Laboratory.
\end{abstract}

\section{Introduction}

The modification of the hadronic properties, i.e., mass and width, of vector mesons (e.g., $\rho, \omega$ and $\phi$ mesons) in a nucleus is an important aspect in the nuclear physics, particularly in context to restore the chiral symmetry of those mesons [1]. The calculated results show the modification of the vector meson in a normal nucleus is significant. The shape of the $\rho$ meson mass distribution spectrum in the pion nucleus reaction is deferred from that in the free space $[2,3]$. The modification of the hadronic parameters is shown to occur for the $\rho$ and $\omega$ mesons produced in the photonuclear reaction $[4,5]$. The in-medium properties of the $\phi$ meson produced in the nuclear reactions are discussed in Ref. [6].

The medium modification of the vector mesons has been explored by measuring the invariant mass distribution spectrum of their decay products. In the heavy-ion collision experiments, the broadening of the $\rho$ meson width in the dielectron and dimuon invariant mass distribution spectra are reported by CERES [7] and NA60 [8] collaborations respectively. The modification of the $\rho, \omega$ and $\phi$ mesons in the proton-nucleus collision has been investigated by the KEK-PS [9] and ANKE collaborations [10]. The previous collaboration has demonstrated the modification in the spectral shape of these mesons due to excess mass on the lower side of the $\omega$ and $\phi$ mesons' peak in the dilepton $e^{+} e^{-}$invariant mass

\footnotetext{
${ }^{1}$ email: swapand@barc.gov.in
} 
distribution spectra, where as the latter collaboration has revealed large spreading in the width of the $\phi$ meson in the dikaon $K^{+} K^{-}$invariant mass distribution spectrum. The CBELSA collaboration $[11,12]$ could not find the mass-shift of the $\omega$ meson, produced in the photonuclear reaction, in the measured $\pi^{0} \gamma$ invariant mass distribution spectrum. This observation is supported by the calculated results [13]. However, the above collaboration has reported the enhancement in the width of the $\omega$ meson $[12,14]$. It should be mentioned that the KEK data (expressing the vector meson mass modification) suffer from the background subtraction which lead to erroneous conclusions, as pointed out by the CLAS collaboration $[15,16]$. This collaboration carried out the systematic investigation of the in-medium properties of the vector mesons (viz., $\rho, \omega$ and $\phi$ mesons) produced in the inclusive photonuclear reaction at Jefferson Laboratory (JLAB). The quoted mesons were detected by their dilepton, i.e., $e^{+} e^{-}$, decay product. The electromagnetic probes, used by the CLAS collaboration, provide the undistorted information about the properties of the vector meson in the nucleus.

The CLAS Collaboration has analyzed the absorption of the $\omega$ and $\phi$ mesons in the nucleus (to search the medium effect on these mesons) by measuring the nuclear transparency ratios vs $A$ (mass number of the nucleus) of the quoted mesons [16]. It should be mentioned that the transparency ratio depends on the elementary vector meson nucleon total scattering cross section $\sigma_{t}^{* V N}$ (i.e., $\sigma_{t}^{* \omega N}$ and $\sigma_{t}^{* \phi N}$ for the considered mesons) in the nucleus. The notation $V$ has been used to express either $\omega$ or $\phi$. The data of the transparency ratio $T_{A} / T_{C}$ (i.e., normalized to ${ }^{12} \mathrm{C}$ ) for the $\omega$ meson could not be reproduced by drastically increasing the free space $\omega$ meson nucleon scattering cross section $\sigma_{t}^{\omega N}$, i.e., $\sigma_{t}^{* \omega N} \geq 10 \sigma_{t}^{\omega N}[16,17]$. On the other hand, $T_{A} / T_{C}$ data for the $\phi$ meson reveal the drastic enhancement of the $\phi$ meson nucleon scattering cross section in the nucleus: $\sigma_{t}^{* \phi N}=(1.5-15) \sigma_{t}^{\phi N}[16,17]$. However, the measured transparency ratios $T_{A} / T_{d}$ (i.e., normalized to deuteron) for both $\omega$ and $\phi$ mesons can be explained by $\sigma_{t}^{* V N} \sim 2 \sigma_{t}^{V N}[17]$. It should be mentioned that the elementary vector meson nucleon cross section in the nucleus is related to the collision broadening of the vector meson, i.e., $\Gamma_{V}^{c} \propto \sigma_{t}^{* V M}$.

The modification of the $\rho$ meson was observed by the CLAS collaboration at JLAB [15] in the measured $e^{+} e^{-}$invariant mass distribution spectra in the photonuclear reaction. This collaboration has reported the enhanced width (not mass-shift) of the $\rho$ meson photoproduced in the nuclei. The calculated results for the quoted spectra also corroborate this finding $[18,19]$. Being a broad resonance, the $\rho$ meson dominantly decays inside the nucleus [18]. Therefore, the invariant mass distribution spectrum of its decay products (e.g., $\rho \rightarrow e^{+} e^{-}$) can elucidate the medium modification of the $\rho$ meson. In contrast to it, the quoted spectrum of the decay products of the narrow resonances (e.g., $\omega$ and $\phi$ mesons) cannot illustrate the in-medium properties of these mesons as they dominantly decay outside the nucleus [13].

The dilepton $e^{+} e^{-}$emission due to the decay of the $\rho$ meson coherently photopro- 
duced in the nucleus had been studied earlier [18], where the nucleus remains in the same (ground) state after the reaction occurred. The calculated $e^{+} e^{-}$invariant mass distribution spectrum in the above reaction shows the significant spreading (without mass-shift) of this meson, specifically, in the heavy nucleus. As mentioned earlier, the CLAS collaboration [15] reported the in-medium properties of the $\rho$ meson by measuring the $e^{+} e^{-}$ invariant mass distribution spectra in the inclusive photonuclear reaction, where the final state of the nucleus is not identified. To analyze this data, the cross sections of the above reaction on nuclei have been calculated, and those are compared with the measured spectra [15].

\section{Formalism}

The dilepton $e^{+} e^{-}$arises due to the decay of the $\rho$ meson produced in the inclusive photonuclear reaction, i.e., $\gamma \mathrm{A} \rightarrow \rho^{0} \mathrm{X} ; \rho^{0} \rightarrow e^{+} e^{-}$. The symbol A represents the nucleus in the initial state, and $\mathrm{X}$ is not specified in the final state. The $\rho$ meson (an unstable particle; $\tau_{\rho} \sim 10^{-23} \mathrm{~s}$ ) propagates certain distance before it decays into dilepton: $\rho \rightarrow$ $e^{+} e^{-}$. The matrix element $\mathcal{M}_{f i}$ for the above reaction can be written as

$$
\mathcal{M}_{f i}=\frac{e \gamma_{\gamma \rho}}{m^{2}}<\phi_{e^{-}}\left(\mathbf{r}^{\prime}\right) \phi_{e^{+}}\left(\mathbf{r}^{\prime}\right) l_{\mu} G_{\rho}^{\mu \nu}\left(\mathbf{r}^{\prime}-\mathbf{r}\right)\left|R_{X 0}(\mathbf{r})\right| \epsilon_{\nu}\left(\mathbf{k}_{\gamma}, \lambda_{\gamma}\right), \phi_{\gamma}(\mathbf{r})>
$$

where $G_{\rho}^{\mu \nu}\left(\mathbf{r}^{\prime}-\mathbf{r}\right)=\left(-g^{\mu \nu}+\frac{k_{\rho}^{\mu} k_{\rho}^{\nu}}{m^{2}}\right) G_{\rho}\left(\mathbf{r}^{\prime}-\mathbf{r}\right)$ is the $\rho$ meson propagator. The second part of it does not contribute to the cross section of the reaction. The scalar part, i.e., $G_{\rho}\left(\mathbf{r}^{\prime}-\mathbf{r}\right)$, describes the $\rho$ meson propagation from $\mathbf{r}$ to $\mathbf{r}^{\prime}$ in the space. The vector meson dominance (VMD) model $[20,21]$ has been used to explain the dielectron decay of the $\rho$ meson. $\gamma_{\gamma \rho}$ denotes the $\rho$ meson to photon (virtual) conversion factor, as illustrated by VMD model. In fact, it is connected to the $\rho$ meson hadron coupling constant $f_{\rho}$ as $\gamma_{\gamma \rho}=\frac{e m_{\rho}^{2}}{f_{\rho}}[20,21]$. The factor $1 / m^{2}$ is the propagator of the virtual photon which coupled to the leptonic current $l_{\mu}=\bar{u}_{e^{-}} \gamma_{\mu} v_{e^{+}}$with the coupling constant $e$ (electronic charge). $\epsilon_{\nu}\left(\mathbf{k}_{\gamma}, \lambda_{\gamma}\right)$ is the polarization four vector of the photon, and $\phi$ s are the wave functions for the continuum particles. $R_{X 0}$ stands for the nuclear transition matrix element: $R_{X 0}(\mathbf{r})=$ $\sum_{j}<X\left|\bar{f}_{\gamma N \rightarrow \rho N}\left(\mathbf{r}-\mathbf{r}_{j}\right)\right| A>$, where $A$ is the initial state (i.e., ground state) of the nucleus, and $X$ represents the unspecified final state. $\bar{f}_{\gamma N \rightarrow \rho N}\left(\mathbf{r}-\mathbf{r}_{j}\right)$ describes the production amplitude of the $\rho$ meson in the elementary $\gamma N \rightarrow \rho N$ reaction occurring on the $j$ th nucleon in the nucleus.

The matrix element $\mathcal{M}_{f i}$ can be decoupled as $\mathcal{M}_{f i}=\Gamma\left(m_{s^{-}}, m_{s^{+}}, \lambda_{\gamma}\right) F_{\rho}(X)$, where the previous factor involves the internal coordinates of the continuum particles, i.e.,

$$
\Gamma\left(m_{s^{-}}, m_{s^{+}}, \lambda_{\gamma}\right)=-\frac{e \gamma_{\gamma \rho}}{m^{2}} \bar{u}\left(k_{e^{-}}, m_{s^{-}}\right) \gamma_{\mu} v\left(k_{e^{+}}, m_{s^{+}}\right) \epsilon^{\mu}\left(k_{\gamma}, \lambda_{\gamma}\right),
$$


and $F_{\rho}(X)$ illustrates the production, propagation and decay of the $\rho$ meson in the space:

$$
F_{\rho}(X)=\iint d \mathbf{r} d \mathbf{r}^{\prime} e^{-i \mathbf{k}_{\rho} \cdot \mathbf{r}^{\prime}} G_{\rho}\left(\mathbf{r}^{\prime}-\mathbf{r}\right) e^{i \mathbf{k}_{\gamma} \cdot \mathbf{r}} R_{X 0}(\mathbf{r}),
$$

with $\mathbf{k}_{\rho}\left(=\mathbf{k}_{e^{+}}+\mathbf{k}_{e^{-}}\right)$expressing the momentum of the $\rho$ meson.

The form for $G_{\rho}\left(\mathbf{r}^{\prime}-\mathbf{r}\right)[2,18]$ is given by

$$
G_{\rho}\left(\mathbf{r}^{\prime}-\mathbf{r}\right)=\delta\left(\mathbf{b}^{\prime}-\mathbf{b}\right) \theta\left(z^{\prime}-z\right) e^{i \mathbf{k}_{\rho} \cdot\left(\mathbf{r}^{\prime}-\mathbf{r}\right)} D_{\mathbf{k}_{\rho}}\left(\mathbf{b}, z^{\prime}, z\right),
$$

where $D_{\mathbf{k}_{\rho}}\left(\mathbf{b}, z^{\prime}, z\right)$ comprises the interaction of the $\rho$ meson with the nucleus:

$$
D_{\mathbf{k}_{\rho}}\left(\mathbf{b}, z^{\prime}, z\right)=-\frac{i}{2 k_{\rho \|}} \exp \left[\frac{i}{2 k_{\rho \|}} \int_{z}^{z^{\prime}} d z^{\prime \prime}\left\{\tilde{G}_{0 \rho}^{-1}(m)-2 E_{\rho} V_{O \rho}\left(\mathbf{b}, z^{\prime \prime}\right)\right\}\right] .
$$

In this equation, $V_{O \rho}$ symbolizes the $\rho$ meson optical potential which modifies the parameters of this meson in the nucleus. $E_{\rho}$ is the energy of the quoted meson. $\tilde{G}_{0 \rho}^{-1}[=$ $\left.m^{2}-m_{\rho}^{2}+i m_{\rho} \Gamma_{\rho}(m)\right]$ represents the inverse of the $\rho$ meson propagator of mass $m$ and total decay width $\Gamma_{\rho}(m)$ in the free space. $m_{\rho}(=775.26 \mathrm{MeV})$ is the pole mass of this meson [22].

Using $G_{\rho}\left(\mathbf{r}^{\prime}-\mathbf{r}\right)$ given in Eq. (4), the expression of $F_{\rho}^{(X)}$ in Eq. (3) can be simplified to

$$
F_{\rho}(X)=\int d \mathbf{r} e^{i \mathbf{q} \cdot \mathbf{r}} D_{k_{\rho}}(\mathbf{b}, z) R_{X 0}(\mathbf{r}),
$$

with $\mathbf{q}\left(=\mathbf{k}_{\gamma}-\mathbf{k}_{\rho}\right)$ being the momentum transfer to the nucleus. $D_{k_{\rho}}(\mathbf{b}, z)$ is given by $D_{k_{\rho}}(\mathbf{b}, z)=\int_{z}^{\infty} d z^{\prime} D_{\mathbf{k}_{\rho}}\left(\mathbf{b}, z^{\prime}, z\right)$.

The differential cross section for the dilepton emission in the inclusive photonuclear reaction can be written as

$$
d \sigma=\frac{\pi^{3}}{(2 \pi)^{8}} \frac{1}{E_{\gamma} E_{e^{-}} E_{e^{+}}} \delta^{4}\left(k_{i}-k_{f}\right)<\left|\mathcal{M}_{f i}\right|^{2}>d \mathbf{k}_{e^{-}} d \mathbf{k}_{e^{+}} d \mathbf{k}_{X},
$$

where $k_{i}\left(k_{f}\right)$ is the four momentum in the initial(final) state of the reaction. $<\left|\mathcal{M}_{f i}\right|^{2}>$ refers to

$$
\begin{aligned}
<\left|\mathcal{M}_{f i}\right|^{2}> & =\frac{1}{2} \sum_{\lambda_{\gamma}} \sum_{m_{s^{-}}, m_{s^{+}}} \sum_{X}\left|\mathcal{M}_{f i}\right|^{2} \\
& =\frac{1}{2} \sum_{\lambda_{\gamma}} \sum_{m_{s^{-}}, m_{s^{+}}}\left|\Gamma\left(m_{s^{-}}, m_{s^{+}}, \lambda_{\gamma}\right)\right|^{2} \sum_{X}\left|F_{\rho}(X)\right|^{2},
\end{aligned}
$$

where $\sum_{X}$ represents the summation over all nuclear final states. $\lambda_{\gamma}$ is the polarization vector of the incoming photon. $m_{s^{-}}$and $m_{s^{+}}$are the spin projections of the outgoing electron and positron respectively. 
Using Eq. (8), the differential cross section for the dilepton $e^{+} e^{-}$invariant mass (i.e., $\rho$ meson mass) distribution in the reaction quoted in Eq. (7) can be expressed as

$$
\frac{d \sigma\left(E_{\gamma}\right)}{d m d \Omega_{\rho}}=K_{F} m^{2} \Gamma(m)_{\rho^{0} \rightarrow e^{+} e^{-}}\left|\bar{f}(0)_{\gamma N \rightarrow \rho N}\right|^{2} \int d \mathbf{r}\left|D_{\mathbf{k}_{\rho}}(\mathbf{b}, z)\right|^{2} \varrho(\mathbf{r}),
$$

with $K_{F}=\frac{3 \pi}{(2 \pi)^{4}} \frac{k_{\rho}^{2}\left(E_{i}-E_{\rho}\right)}{E_{\gamma}\left|k_{\rho} E_{i}-\mathbf{k}_{\gamma} \cdot \hat{k}_{\rho} E_{\rho}\right|} . \quad \Gamma(m)_{\rho^{0} \rightarrow e^{+} e^{-}}$is the width of the $\rho$ meson of mass $m$ decaying at rest into $e^{+} e^{-}$.

The differential cross section stated in Eq. (9) is based on the fixed scatterer or frozen nucleon (in the nucleus) approximation. The Fermi motion of the nucleon in the nucleus can be incorporated replacing $\bar{f}_{\gamma N \rightarrow \rho N}$ by $<\bar{f}_{\gamma N \rightarrow \rho N}>_{A}$, i.e.,

$$
<\bar{f}(0)_{\gamma N \rightarrow \rho N}>_{A}=\iint d \mathbf{k}_{N} d \epsilon_{N} S_{A}\left(\mathbf{k}_{N}, \epsilon_{N}\right) \bar{f}(0, s)_{\gamma N \rightarrow \rho N}
$$

with

$$
\begin{aligned}
s & =\left(E_{\gamma}+E_{N}\right)^{2}-\left(\mathbf{k}_{\gamma}+\mathbf{k}_{N}\right)^{2} \\
E_{N} & =m_{A}-\sqrt{k_{N}^{2}+\left(m_{A}-m_{N}+\epsilon_{N}\right)^{2}},
\end{aligned}
$$

where $S_{A}\left(\mathbf{k}_{N}, \epsilon_{N}\right)$ represents the spectral function of the nucleus, normalized to unity. It illustrates the probability of a nucleon with momentum $\mathbf{k}_{N}$ and binding energy $\epsilon_{N}$ in the nucleus [23]. $S_{A}\left(\mathbf{k}_{N}, \epsilon_{N}\right)$ for various nuclei are discussed elaborately in Ref. [24]. Therefore, those have not been presented explicitly.

It should be mentioned that the tagged photon beam was used for the experiment done by the CLAS collaboration at Jlab [15]. The quoted beam possesses certain energy range which can be weighted in 6 bins to simulate the beam profile [19] (also see the references there in). Therefore, the cross section of the considered reaction due to tagged $\gamma$ beam can be written [18] as

$$
\frac{d \sigma}{d m}=\sum_{i=1}^{6} W\left(E_{\gamma, i}\right) \frac{d \sigma\left(E_{\gamma, i}\right)}{d m}
$$

where $W\left(E_{\gamma, i}\right)$ is the relative weights of $13.7 \%, 23.5 \%, 19.3 \%, 20.1 \%, 12.6 \%$ and $10.9 \%$ for $E_{\gamma, i}(\mathrm{GeV})$ equal to $1.0,1.5,2.0,2.5,3.0$ and 3.5 respectively [19].

\section{$3 \quad$ Result and Discussions}

The total decay width $\Gamma_{\rho}(m)$ of the $\rho$ meson in the free space is composed of the partial widths of the $\rho$ meson decaying into various channels, i.e., $\Gamma_{\rho}(m) \approx 99.94 \times$ 
$10^{-2} \Gamma(m)_{\rho \rightarrow \pi^{+} \pi^{-}}+6 \times 10^{-4} \Gamma(m)_{\rho \rightarrow \pi^{0} \gamma}[22]$. The two body decay width of the $\rho$ meson $\Gamma(m)_{\rho \rightarrow d_{1} d_{2}}[25]$ is given by

$$
\Gamma(m)_{\rho \rightarrow d_{1} d_{2}}=\Gamma\left(m_{\rho}\right)_{\rho \rightarrow d_{1} d_{2}}\left[\frac{\Phi_{l}(m)}{\Phi_{l}\left(m_{\rho}\right)}\right] .
$$

The values of $\Gamma\left(m_{\rho}\right)_{\rho \rightarrow \pi^{+} \pi^{-}}$and $\Gamma\left(m_{\rho}\right)_{\rho \rightarrow \pi^{0} \gamma}$ are tabulated in Ref. [22]. $\Phi_{l}$ represents the phase space factor of the two body decay: $\Phi_{l}(m)=\frac{\tilde{k}}{m} B_{l}^{2}(\tilde{k} R)$, with the interaction radius $R=1 \mathrm{fm}[4] . \tilde{k}$ is the momentum in the cm system of the decay products of the $\rho$ meson. The angular momentum $l$ associated with the considered $\rho$ meson decay channels is equal to unity. $B_{l}^{2}(\tilde{k} R)$ represents the Blatt-Weisskopf barrier penetration factor: $B_{1}^{2}(\mathrm{x})=\frac{\mathrm{x}^{2}}{1+\mathrm{X}^{2}}$ [25]. The dielectron decay width of the $\rho$ meson, negligibly small compared to $\Gamma_{\rho}(m)$, used in Eq. (9) is given by $\Gamma(m)_{\rho^{0} \rightarrow e^{+} e^{-}}=\frac{\pi}{3}\left(\frac{\alpha_{e m}}{\gamma_{\rho}}\right)^{2} \frac{m_{\rho}^{4}}{m^{3}}[21] . \alpha_{e m}(=1 / 137.04)$ denotes the fine structure constant, and $\gamma_{\rho}$ is equal to half of the $\rho$ meson hadron coupling constant $f_{\rho}\left(=2 \gamma_{\rho}\right)$, as mentioned earlier. The value of $\gamma_{\rho}(=2.48[26])$ is directly determined from the measured $\Gamma\left(m_{\rho}\right)_{\rho \rightarrow e^{+} e^{-}}[22]$.

The $\rho$ meson production amplitude for the elementary $\gamma N \rightarrow \rho N$ reaction can be expressed as $\bar{f}_{\gamma N \rightarrow \rho N}=-4 \pi E_{\rho}\left[\frac{1}{\tilde{E}_{\rho}}+\frac{1}{\tilde{E}_{N}}\right] \tilde{f}_{\gamma N \rightarrow \rho N}$ [27]. The symbol " " on the quantities represents those assessed at $\gamma N$ cm energy. $f_{\gamma N \rightarrow \rho N}$ denotes the reaction amplitude of the quoted elementary reaction. The vector meson dominance model connects $f_{\gamma N \rightarrow \rho N}$ to the $\rho N \rightarrow \rho N$ scattering amplitude $f_{\rho N \rightarrow \rho N}$ as $f_{\gamma N \rightarrow \rho N}=\frac{\sqrt{\pi \alpha_{e m}}}{\gamma_{\rho}} f_{\rho N \rightarrow \rho N}[26,28]$. The energy dependent experimentally determined values of the forward $f_{\rho N \rightarrow \rho N}$ are given in Ref. [29].

The optical potential of the $\rho$ meson $V_{O \rho}$, which describes the $\rho$ meson nucleus interaction, can be expressed [30] as

$$
V_{O \rho}(\mathbf{r})=-\frac{v_{\rho}}{2}\left(\alpha_{\rho N}+i\right) \sigma_{t}^{\rho N} \varrho(\mathbf{r})
$$

where $v_{\rho}$ is the velocity of the $\rho$ meson. $\alpha_{\rho N}$ represents the ratio of the real to imaginary part of $f_{\rho N \rightarrow \rho N}(0)$ in the free space. $\sigma_{t}^{\rho N}$ denotes the total $\rho$ meson nucleon scattering cross section: $\sigma_{t}^{\rho N}=\frac{4 \pi}{k_{\rho}} f_{\rho N \rightarrow \rho N}(0) . \varrho(\mathbf{r})$ symbolizes the density distribution of the nucleus, normalized to the mass number of the nucleus. The form for it, as extracted from the electron scattering data [31], is used to evaluate $V_{O \rho}(\mathbf{r})$.

The differential cross sections $\frac{d \sigma}{d m}$ of the $\rho$ meson mass $m$ (i.e., $e^{+} e^{-}$invariant mass) distribution in the inclusive photonuclear reaction, i.e., $\mathrm{A}\left(\gamma, \rho \rightarrow e^{+} e^{-}\right) \mathrm{X}$, have been calculated for the $\rho$ meson momentum $k_{\rho}=0.8-3.0 \mathrm{GeV} / c$, as that was the restriction on $k_{\rho}$ imposed in the CLAS measurements [15]. The calculated $\frac{d \sigma}{d m}$ for ${ }^{12} \mathrm{C}$ and ${ }^{56} \mathrm{Fe}$ nuclei (normalized to the experimental counts for $\mathrm{C}$ and Fe-Ti nuclei [15] respectively) have been presented in Fig. 1 for the $\rho$ meson pole mass $m_{\rho}$ taken equal to $750.26 \mathrm{MeV}$ 
(solid curve) and $775.26 \mathrm{MeV}$ (dot-dashed curve). As visible in this figure, the reduction of $m_{\rho}$ from $775.26 \mathrm{MeV}$ to $750.26 \mathrm{MeV}(\sim 3 \%)$ shows good agreement of the calculated results with the measured spectra for all nuclei. It is remarkable that the quoted reduction of $m_{\rho}$ is consistence with the mass-shift parameter $\left(\alpha_{\rho}=0.02 \pm 0.02\right)$ reported by the CLAS collaboration [15]. Therefore, $m_{\rho}$ is taken equal to $750.26 \mathrm{MeV}$ to investigate the modification of the $\rho$ meson parameters in the nucleus.

The cross sections $\frac{d \sigma}{d m}$ with and without incorporating the $\rho$ meson optical potential $V_{O \rho}$ are calculated to disentangle the nuclear effects on the $\rho$ meson. The calculated results for ${ }^{12} \mathrm{C},{ }^{56} \mathrm{Fe}$ and ${ }^{208} \mathrm{~Pb}$ nuclei are depicted in Fig. 2. The dot-dot-dashed curve illustrates $\frac{d \sigma}{d m}$ assessed without $V_{O \rho}$ and the solid curve represents that evaluated including $V_{O \rho}$ in the calculation. This figure distinctly elucidates the broadening in the width of the $\rho$ meson mass distribution spectra due to $V_{O \rho}$ for all nuclei without the shift in the peak position, i.e., mass-shift. The quantitative values of the widths of the $\rho$ meson mass distribution spectra $\Gamma_{\rho}^{*}$, and the enhancement in the widths $\Delta \Gamma_{\rho}$ due to $V_{O \rho}$ for the above mentioned nuclei are listed in table 1.

The calculated $\frac{d \sigma}{d m}$ are compared in Fig. 3 for ${ }^{12} \mathrm{C}$ (dot-dot-dashed curve), ${ }^{56} \mathrm{Fe}$ (dashed curve) and ${ }^{208} \mathrm{~Pb}$ (dot-dashed curve) nuclei to visualize the relative broadening in the $\rho$ meson mass distribution spectra. It is noticeable in the figure that the spreading of the spectrum increases with the size of the nucleus. Therefore, the medium modification of the $\rho$ meson properties can be demonstrated better in the heavy nucleus.

Table 1: The calculated width of the $\rho$ meson mass distribution spectrum $\Gamma_{\rho}^{*}$, and the enhancement in the width $\Delta \Gamma_{\rho}$ due to the $\rho$ meson nucleus interaction $V_{O \rho}$.

\begin{tabular}{ccc}
\hline Nucleus & $\Gamma_{\rho}^{*}(\mathrm{MeV})$ & $\Delta \Gamma_{\rho}(\mathrm{MeV})$ \\
\hline${ }^{12} \mathrm{C}$ & 163.5 & 19.6 \\
${ }^{56} \mathrm{Fe}$ & 172.1 & 29.4 \\
${ }^{208} \mathrm{~Pb}$ & 197.5 & 54.8 \\
\hline
\end{tabular}

\section{Conclusions}

The differential cross section for the $\rho$ meson mass distribution in the inclusive photonuclear reaction has been calculated to investigate the modification of the hadronic parameters (i.e., mass and width) of the $\rho$ meson in the nucleus. The quoted modification arises due to the interaction of the $\rho$ meson with the nucleus, which is described by the $\rho$ meson optical potential. It is evaluated by folding the $\rho$ meson nucleon scattering amplitude with the density distribution of the nucleus. The calculated result does not show the mass modification in the $\rho$ meson mass distribution spectrum, but that illustrates the 
increase in the width of the quoted spectrum. The enhancement in the width increases with the mass of the nucleus. The calculated $\rho$ meson mass distribution spectra for nuclei reproduce well the data reported from the Jefferson Laboratory.

\section{Acknowledgement}

The author appreciates Dr. S. M. Yusuf for the support and encouragement to work on theoretical nuclear physics. 


\section{Figure Captions}

1. (color online). The differential cross sections $\frac{d \sigma}{d m}$ calculated for the $\rho$ meson mass distribution in the inclusive photoinduced reactions on ${ }^{12} \mathrm{C}$ and ${ }^{56} \mathrm{Fe}$ are compared with data taken for $\mathrm{C}$ and Fe-Ti nuclei [15] respectively. The calculated results are normalized to the experimental counts.

2. (color online). The $\rho$ meson mass distribution spectra calculated with and without the $\rho$ meson optical potential $V_{O \rho}$ are presented for nuclei. The broadening in the width (not peak-shift) of the spectrum due to $V_{O \rho}$ is distinctly visible in the figure.

3. (color online). The $\rho$ meson mass distribution spectra for ${ }^{12} \mathrm{C},{ }^{56} \mathrm{Fe}$, and ${ }^{208} \mathrm{~Pb}$ nuclei are compared. The enhancement in the width of the spectrum due to $V_{O \rho}$ increases with the size of the nucleus. 


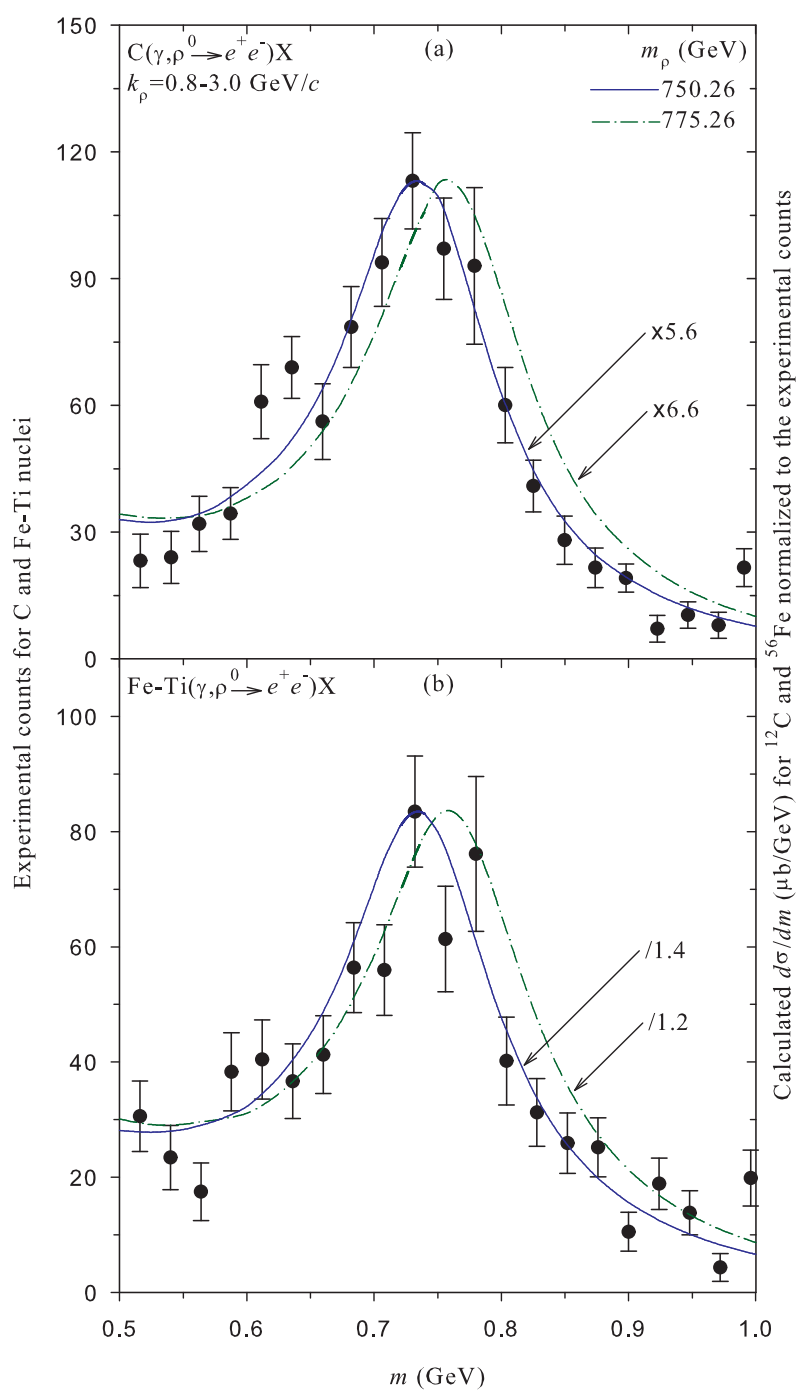

Figure 1: (color online). The differential cross sections $\frac{d \sigma}{d m}$ calculated for the $\rho$ meson mass distribution in the inclusive photoinduced reactions on ${ }^{12} \mathrm{C}$ and ${ }^{56} \mathrm{Fe}$ are compared with data taken for $\mathrm{C}$ and Fe-Ti nuclei [15] respectively. The calculated results are normalized to the experimental counts. 


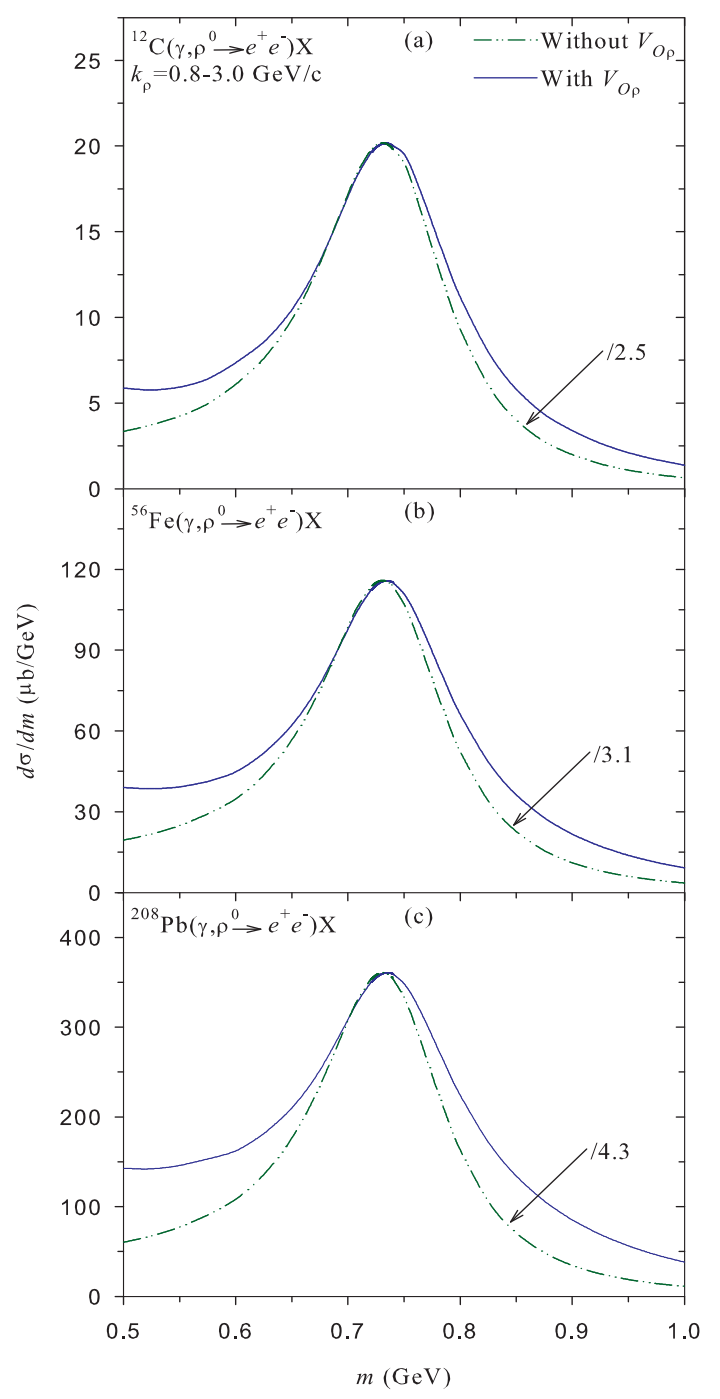

Figure 2: (color online). The $\rho$ meson mass distribution spectra calculated with and without the $\rho$ meson optical potential $V_{O \rho}$ are presented for nuclei. The broadening in the width (not peak-shift) of the spectrum due to $V_{O \rho}$ is distinctly visible in the figure. 


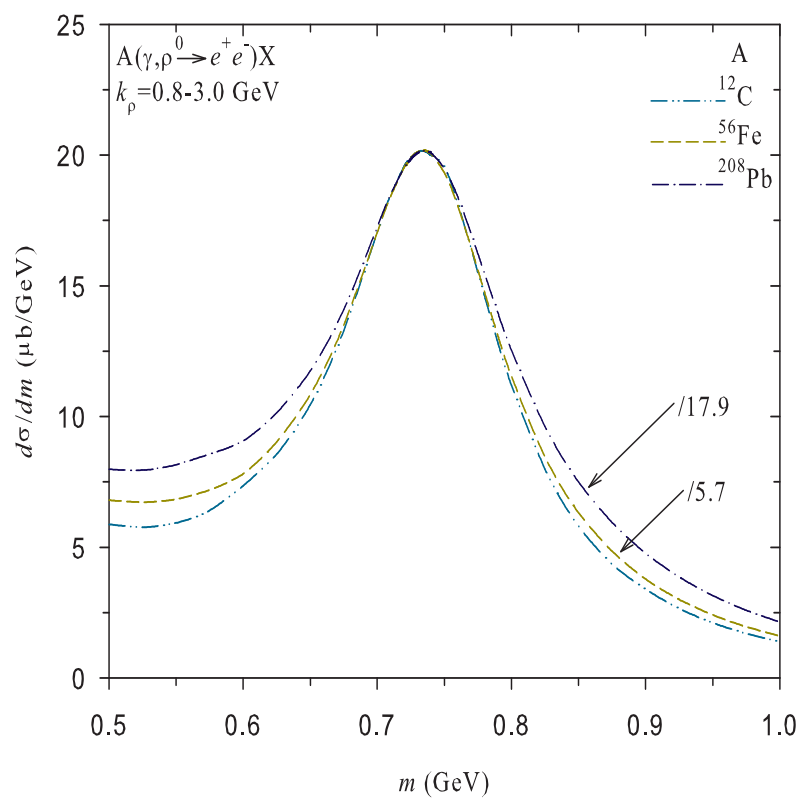

Figure 3: (color online). The $\rho$ meson mass distribution spectra for ${ }^{12} \mathrm{C},{ }^{56} \mathrm{Fe}$, and ${ }^{208} \mathrm{~Pb}$ nuclei are compared. The enhancement in the width of the spectrum due to $V_{O \rho}$ increases with the size of the nucleus. 


\section{References}

[1] G. E. Brown and M. Rho, Phys. Rev. Lett. 66 (1991) 2720; T. Hatsuda and S. H. Lee, Phys. Rev. C 46 (1992) R34.

[2] Ye. S. Golubeva, L. A. Kondratyuk, and W. Cassing, Nucl. Phys. A 625 (1997) 832.

[3] Th. Weidmann, E. L. Bratkovskaya, W. Cassing, and U. Mosel, Phys. Rev. C 59 (1999) 919; M. Effenberger, E. L. Bratkovskaya, W. Cassing, and U. Mosel, ibid. 60 (1999) 027601.

[4] M. Effenberger, E. L. Bratkovskaya, and U. Mosel, Phys. Rev. C 60 (1999) 044614.

[5] P. Mühlich and U. Mosel, Nucl. Phys. A 773 (2006) 156; P. Mühlich, V. Shklyar, S. Leupold, U. Mosel and M. Post, Nucl. Phys. A 780 (2006) 187.

[6] D. Cabrera, L. Roca, E. Oset, H. Toki, and M. J. Vicente Vacas, Nucl. Phys. A 733 (2004) 130; V. K. Magas, L. Roca, and E. Oset, Phys. Rev. C 71 (2005) 065202.

[7] D. Adamová et al., (CERES Collaboration), Phys. Lett. B 666 (2008) 425.

[8] R. Arnaldi et al., (NA60 Collaboration), Phys. Rev. Lett. 96 (2006) 162302; Eur. Phys. J. C 49 (2007) 235; S. Damjanovic et al., (NA60 Collaboration), Nucl. Phys. A 783 (2007) 327c.

[9] M. Naruki et al., Phys. Rev. Lett. 96 (2006) 092301; R. Muto et al., (KEK-PS E325 Collaboration), Phys. Rev. Lett. 98 (2007) 042501.

[10] A. Polyanskiy et al., Phys. Lett. B 695 (2011) 74; M. Hartmann et al., Phys. Rev. C 85 (2012) 035206.

[11] M. Nanova et al., (CBELSA/TAPS Collaboration), Phys. Rev. C 82 (2010) 035209; Eur. Phys. J. A 47 (2011) 16.

[12] M. Thiel et al., (CBELSA/TAPS Collaboration), Eur. Phys. J. A 49 (2013) 132.

[13] S. Das, Phys. Rev. C 83 (2011) 064608.

[14] M. Kotulla et al., (CBELSA/TAPS Collaboration), Phys. Rev. Lett. 100 (2008) 192302; erratum, 114 (2015) 199903; S. Friedrich et al., (CBELSA/TAPS Collaboration), Eur. Phys. J. A 52 (2016) 297.

[15] R. Nasseripour et al., (CLAS Collaboration), Phys. Rev. Lett. 99 (2007) 262302; M. H. Wood et al., (CLAS Collaboration), Phys. Rev. C 78 (2008) 015201. 
[16] M. H. Wood et al, (CLAS Collaboration), Phys. Rev. Lett. 105 (2010) 112301.

[17] S. Das, Eur. Phys. J. A 55 (2019) 186.

[18] S. Das, Eur. Phys. J. A 49 (2013) 123.

[19] F. Riek, R. Rapp, T.-S. H. Lee and Y. Oh, Phys. Lett. B 677 (2009) 116; Phys. Rev. C 82 (2010) 015202.

[20] J. J. Sakurai, Currents and Mesons (The University of Chicago Press, Chicago, 1969). p. 48; R. K. Bhaduri, Models of the Nucleon (Addison-Wesley Publishing Company, Inc, California, 1988), p. 261.

[21] G. Q. Li, C. M. Ko, G. E. Brown and H. Sorge, Nucl. Phys. A 611 (1996) 539.

[22] M. Tanabashi, et al., (Particle Data Group), Phys. Rev. D 98 (2018) 030001; http://pdg.lbl.gov./xsect/contents.html.

[23] E. Ya. Paryev, J. Phys. G 40 (2013) 025201.

[24] E. Ya. Paryev, Eur. Phys. J. A 7 (2000) 127.

[25] D. M. Manley and E. M. Saleski, Phys. Rev. D 45 (1992) 4002; D. M. Manley, R. A. Arndt, Y. Goradia, and V. L. Teplitz, ibid. 30 (1984) 904; D. M. Manley, ibid. 51 (1995) 4837; Int. J. Mod. Phys. A 18 (2003) 441.

[26] S. Das, Phys. Rev. C 96 (2017) 034616; A. Sibirtsev, H.-W. Hammer, U.-G. Meißner and A. W. Thomas, Eur. Phys. J. A 29 (2006) 209.

[27] S. Das, Phys. Rev. C 94 (2016) 025204; A. Pautz and G. Shaw, Phys. Rev. C 57 (1998) 2648; L. Frankfurt, M. Strikman and M. Zhalov, Phys. Rev. C 67 (2003) 034901.

[28] T. H. Bauer, R. D. Spital, D. R. Yennie and F. M. Pipkin, Rev. Mod. Phys. 50 (1978) 261; Erratum, ibid. 51 (1979) 407.

[29] L. A. Kondratyuk, A. Sibirtsev, W. Cassing, Ye. S. Golubeva and M. Effenberger, Phys. Rev. C 58 (1998) 1078.

[30] R. J. Glauber, in Lectures in Theoretical Physics, Vol. 1, edited by W. E. Brittin et al., (Interscience, New York, 1959), p. 315; J. M. Eisenberg and D. S. Kolton, Theory of Meson Interaction with Nuclei (John Wiley \& Sons, New York, 1980), p. 158. 
[31] C. W. De Jager, H. De Vries and C. De Vries, At. Data Nucl. Data Tables, 14 (1974) 479. 